\title{
Towards Touchless Pore Fingerprint Biometrics: A Neural Approach
}

\author{
Angelo Genovese, Enrique Muñoz, Vincenzo Piuri, Fabio Scotti, Gianluca Sforza \\ Department of Computer Science, Università degli Studi di Milano, Italy. \\ firstname.lastname@unimi.it
}

\begin{abstract}
Touchless fingerprint recognition systems are being increasingly used for a fast, hygienic, and distortion-free recognition. However, due to the greater complexity of the algorithms required for processing touchless fingerprint samples, currently only Level 1 and Level 2 features are being used for recognition, and Level 3 features are used only in touch-based optical devices with about 1000 ppi resolution. In this paper, we propose the first innovative method in the literature able to extract Level 3 features, in particular sweat pores, from fingerprint images captured with a touchless acquisition using a commercial offthe-shelf camera. The method uses image processing algorithms to extract a set of candidate sweat pores. Then, computational intelligence techniques based on neural networks are used to learn the local features of the real pores, and select only the actual sweat pores from the set of candidate points. The results show the validity of the proposed methodology, with the majority of the pores correctly extracted, indicating that a touchless fingerprint recognition using Level 3 features is feasible.
\end{abstract}

\section{INTRODUCTION}

Fingerprint recognition systems are based on features extracted with three possible levels of detail, with increasing spatial resolution required for their capture, commonly referred as Level 1, Level 2, or Level 3 features. In particular, Level 1 features are related to the general ridge flow and to the position and type of singular points [1]. Level 2 features are based on the position and orientation of local ridge characteristics, called minutiae points, and require at least a 500 ppi resolution [2]. Level 3 features describe intra-ridge characteristics, such as incipient ridges or sweat pores, and require about 700-1000 ppi resolution [3]. Currently, Level 2 features are the most used for recognition, and optical touch-based devices with the required 500 ppi resolution can be found in many scenarios, from government applications (e.g., border control [4]-[6]) to personal devices (e.g., laptops, smartphones [7]).

However, in the last years, touchless fingerprint recognition approaches have been increasingly proposed in the literature and in industrial applications since they do not require the contact of the finger with a surface, thus preventing distortions, hygiene problems, and therefore increasing the social acceptance of biometric technologies. Moreover, they allow a fast recognition, because it is not necessary to wait for the finger

This work was supported in part by: the EC within the 7FP under grant agreement 312797 (ABC4EU); the EC within the $\mathrm{H} 2020$ program under grant agreement 644597 (ESCUDO-CLOUD); and the Italian Ministry of Research within the project "GenData 2020" (2010RTFWBH). The authors thank Dr R. Donida Labati for the fruitful discussions and valuable suggestions as well as for the support in the system design and deployment. to be placed on the sensor with the correct amount of pressure, as happens in the case of touch-based devices. In particular, both two-dimensional [8]-[12] and three-dimensional [13][20] acquisition systems have been described, with results in some cases comparable to optical touch-based systems [20]. Recently, due to the increasing quality of CMOS sensors in portable mobile devices, numerous approaches have also been designed for a touchless fingerprint recognition using smartphones [21]-[23].

In this context, touchless fingerprint recognition approaches require more complex processing algorithms in order to compensate for the non-idealities introduced by a less-constrained acquisition procedure, such as out-of-focus, motion blur, or a complex background. For these reasons, currently only Level 1 or Level 2 features have been used for recognition [16], and no approach has been yet proposed for a touchless fingerprint recognition using Level 3 features. The use of such features could help in implementing liveness-based antispoofing methods [24] and in improving the recognition accuracy in touchless fingerprint recognition systems. In some cases, a reduction in the EER up to $20 \%$ has been obtained by combining Level 1, Level 2, and Level 3 features [25].

In this paper, we propose a novel method for the extraction of the positions of sweat pores (Figure 1), which are the most distinctive Level 3 features [2], from touchless fingerprint images captured with a commercial camera. The proposed approach is a feasibility study towards the use of Level 3 features in touchless fingerprint recognition systems, and the description of algorithms for biometric recognition is out of the scope of the paper. The approach is based on an innovative algorithm for the extraction of Level 3 features in touchless fingerprint images, composed of a novel sequence of steps using standard image processing and computational intelligence techniques. In particular, since the characteristics of the pores may change in different fingerprints, or in different positions of the fingerprint itself according to illumination and wear, computational intelligence methods are used to learn the local features of the real pores, adapt to the operational and environmental conditions, and perform an accurate extraction. To the best of our knowledge, this is the first method in the literature able to extract the position of pores in touchless fingerprint images. We evaluated the proposed approach on a database composed by 66 images captured from 12 persons. In order to establish the ground truth, the pores in each image were manually labeled by three supervisors, for a total of 9,143 pores. The results showed that a touchless fingerprint 


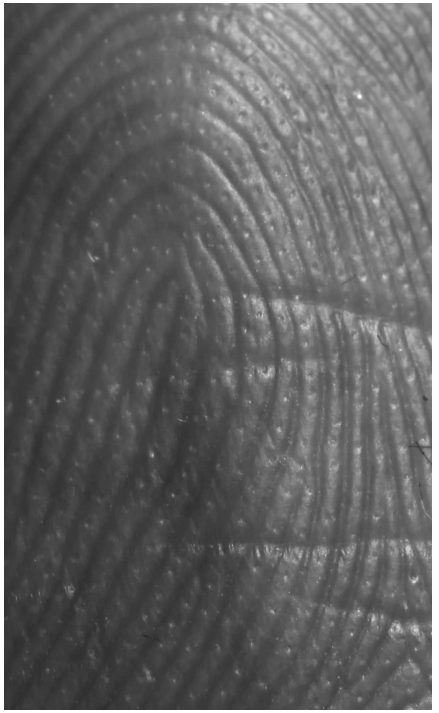

(a)

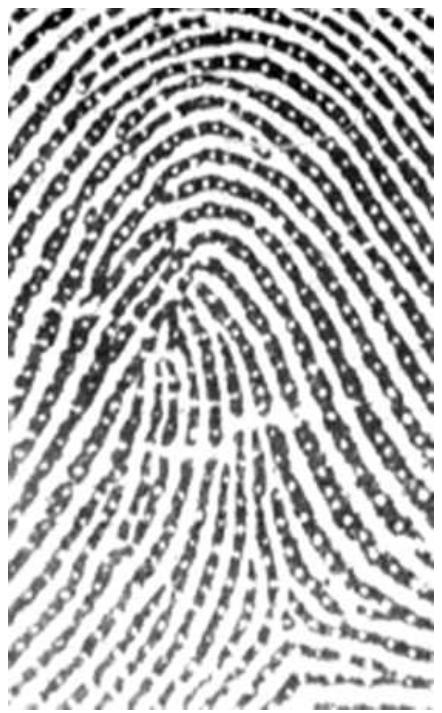

(b)
Fig. 1. Particulars of a touchless fingerprint image captured using the proposed method and a touch-based image captured using an optical device: (a) touchless image; (b) touch-based image. It is possible to observe the sweat pores, which are the most significant Level 3 features, present on the ridges. However, the touchless image exhibits a minor contrast between the pores and ridges, resulting in the necessity of more complex processing algorithms.

recognition system using Level 3 feature is feasible.

The paper is structured as follows: Section II presents a literature review, while Section III describes the proposed methodology. Section IV and Section V contain the experimental results and the conclusions, respectively.

\section{RELATED WORKS}

Currently, no approach in literature has been proposed for the extraction of Level 3 features from touchless fingerprint images. However, several approaches describe the use in touch-based images of Level 3 features, in particular sweat pores, for the purposes of recognition, antispoofing, quality estimation, and image preprocessing.

For the purpose of recognition, methods for extracting the positions of the pores use Gabor Filters [25], wavelet transforms [25], [26], watershed segmentation [27], or morphological operators [28]. Matching methods specifically designed for pores use ICP [25], RANSAC [29], local binary patterns [30], Delaunay triangulation [26], or local features [29], [31]. Level 3 features can also increase the accuracy of fingerprint recognition in the case of partial images [32]-[34] or rolled ink images [35].

Furthermore, antispoofing methods based on Level 3 features have been recently gaining popularity, since the position and size of the pores is more difficult to counterfeit, with respect to the ridge structure and the minutiae [36]. In this context, several methods use statistical features [37]-[39], NIST NFIQ quality indexes [37], [40], Euclidean distance between pores [40], or the number of pores [37], [40], [41].

Lastly, some methods propose Level 3 features for the quality estimation of fingerprint images [42], [43], along with methods that use the position of the pores for image preprocessing [44].

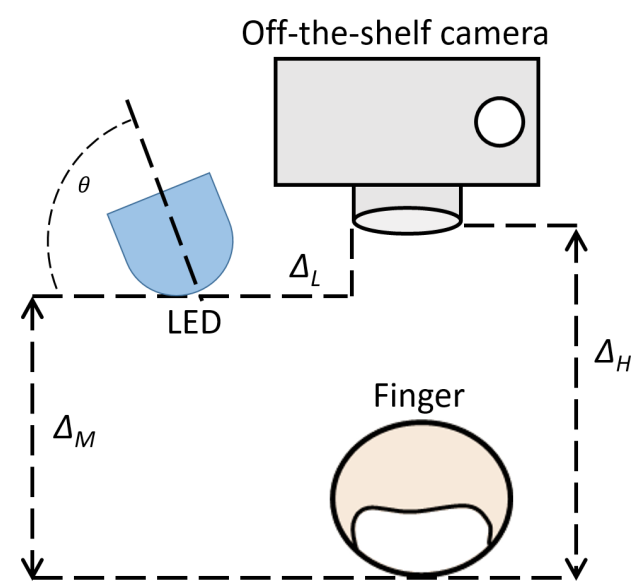

Fig. 2. Schema of the used acquisition setup.

\section{PROPOSED APPROACH}

The proposed approach has the purpose of extracting Level 3 features, in particular the position of the sweat pores, from touchless fingerprint images captured using a commercial camera. Such features could be used for multiple purposes in the field of touchless fingerprint recognition systems, such as increasing the recognition accuracy and implementing antispoofing methods. Our method is based on a single touchless acquisition, and we use an innovative algorithm for the extraction of Level 3 features in touchless fingerprint images, composed of a novel sequence of steps using standard image processing and computational intelligence techniques. In particular, image processing algorithms are used to extract a set of candidate sweat pores, then computational intelligence techniques are used to learn the local features of the real pores, adapt to differences in the illumination and wear of the finger, and select only the actual sweat pores from the set of candidate points.

In particular, the method is composed by the following steps: i) acquisition; ii) extraction of candidate points; iii) neural post-processing.

\section{A. Acquisition}

The acquisition is performed by placing the fingertip on a flat surface and in the field of view of the camera (Figure 2). No support for the finger is used, and the only requirement is that the finger is focused and placed inside the field of view of the camera. A single touchless acquisition is then performed, and the image $I$ is captured. We used a LED illumination to enhance the details of the fingerprint, so that the sweat pores are visible in the captured image. A green LED was used since shorter wavelengths are less absorbed by the skin and result in more contrasted images [45]. However, while a blue wavelength is shorter and would be even less absorbed with respect to the green one, blue LEDs have a smaller light intensity and would require too high shutter times in order to avoid motion blur. 


\section{B. Extraction of candidate points}

The design of the algorithm for the extraction of the candidate pore points is based on two main observations: i) the finger is not uniformly illuminated in the picture; ii) reflections are present on the boundaries of several pores. Then, in order to detect the pores, we exploited the presence of reflections in correspondence of the pores. Moreover, to tackle the non-uniformity of the light, we used the gamma transform for analyzing the image at various contrast conditions, and investigate different details of the image each time.

To identify possible candidates, our algorithm performs the following steps:

1) selection of the green channel $G$ from the input RGB image $I$;

2) gamma transform of the image $G$ at powers $\gamma=$ $\{0.67,1.0,1.5,2.5,5.0\}$, obtaining $G_{\gamma}$;

3) for each gamma image $G_{\gamma}$ :

a) min-max normalization, specifying the bottom $1 \%$ and the top $1 \%$ of all pixel values as the min and max, respectively;

b) median filtering with radius $r_{m}=3$ pixels, to reduce noise while preserving edges, obtaining the image $G_{\gamma, f}$;

c) Sobel edge detection and binarization, obtaining the image $G_{\gamma, b}$

d) close operation applied on $G_{\gamma, b}$ with a circular structuring element of radius $r_{c}=2$ pixels;

e) extraction of the connected components $c_{\gamma, n}$ with area $100 \leq A \leq 900$ and eccentricity $E \leq 0.9$ from $G_{\gamma, b}$, obtaining the binary image $B_{\gamma}$;

f) computation of the coordinates of the centroid $\left(C_{x}, C_{y}\right)_{\gamma, n}$ for every selected component $c_{\gamma, n}$;

4) OR composition of every mask $B_{\gamma}$, produced in the step 3 from the corresponding gamma image $G_{\gamma}$, into a single mask $B$.

We quantified empirically the optimal values of the parameters $\gamma, A$, and $E$ based on the physical properties of the images. The result is a binary image that includes the connected components $c_{n}$, with $1 \leq n \leq N$, where $N$ is the number of candidate pores. The centroids of the connected components $\left(C_{x}, C_{y}\right)_{n}$ are then used to construct the set of $N$ candidate pore points $P C=\left\{\left(C_{x}, C_{y}\right)_{n}\right\}$. Figure 3 presents the output of the candidate extraction algorithm on a sample image.

\section{Neural post processing}

To determine if a candidate point is a real pore or not, we use neural network classifiers. In fact, neural techniques are used to learn the local features of the real pores, adapt to differences in the illumination and wear of the finger, and select only the actual sweat pores from the set of candidate points. In particular, the proposed approach extracts different sets of features that represent different characteristics of the candidate point $P C_{n}$, and uses them to train a neural network classifier. In particular, we distinguish two types of characteristics: the first describes features of the estimated

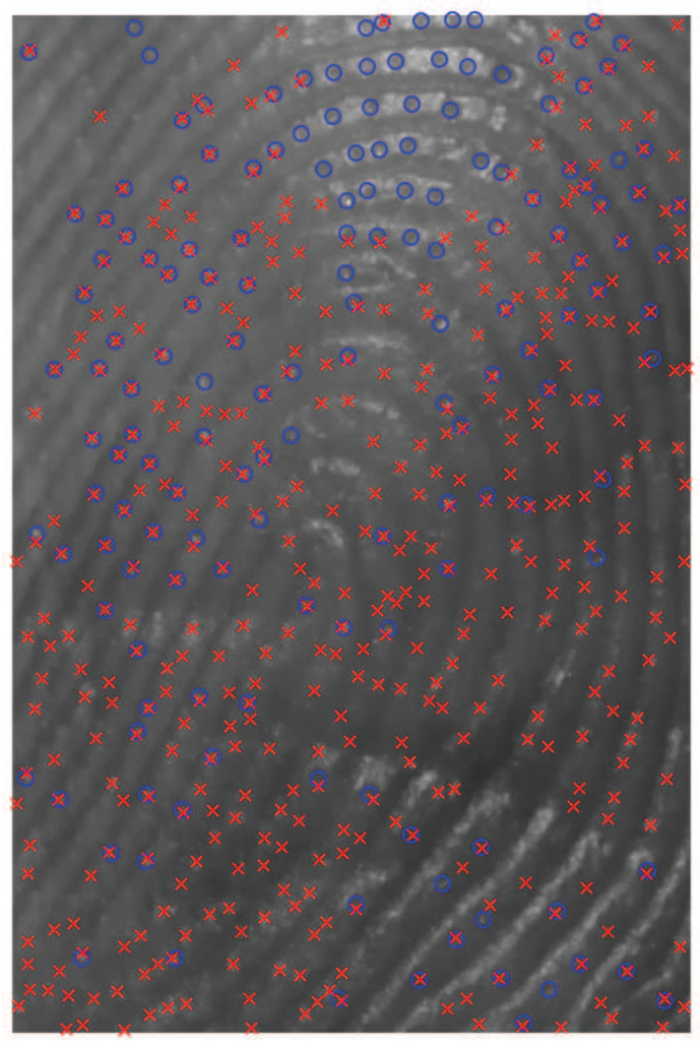

Fig. 3. Output of the candidate extraction step: blue circles mark the manually found pores, while red crosses mark the candidates found by the algorithm. Candidate points are then used in the neural post-processing step in order to select the actual pores.

segmentation region $c_{n}$ (intended as the binary mask that represents it), the second describes characteristics of a portion of the image $I_{n}$ that surrounds the candidate point. This separation is necessary because some features obtain better results with a gray-scale image to discriminate between the candidate points that represent a pore and those that do not represent it.

The first type of features, related to the estimated segmentation, include:

- Area $\left(F_{A}\right)$ : the number of pixels included in the segmented region;

- Convex area $\left(F_{C A}\right)$ : the area, in pixels, of the smallest convex polygon, $c p$, that can contain the segmented region;

- Coordinates $\left(F_{C o}\right)$ : the coordinates of the centroid of the estimated segmentation;

- Equivalent diameter $\left(F_{E D}\right)$ : the diameter of the circle that has the same area as the region;

- Euler number $\left(F_{E N}\right)$ : the inverse of the number of holes in the segmented region;

- Extent $\left(F_{E x}\right)$ : the ratio of the area of the segmented region and the area of the smallest rectangle containing it;

- Filled area $\left(F_{F A}\right)$ : the area, in pixels, of the segmented region when all the holes in it have been filled;

- Major and minor axis length: the length, measured in pixels, of the major and minor axis of the ellipse, $e$, whose 
normalized second central moments correspond to those of the segmented region;

- Eccentricity $\left(F_{E c}\right)$ : the eccentricity of $e$;

- Orientation $\left(F_{O r}\right)$ : the angle between the $\mathrm{x}$-axis and the major axis of $e$;

- Perimeter $\left(F_{P}\right)$ : the number of pixels round the boundary of the segmented region;

- Solidity $\left(F_{S}\right)$ : the proportion of the pixels in $c p$ that are also in the region;

The second type of features is related to the portion of the image $I_{n}$, with size $30 \times 30$ pixels, that surrounds the candidate pore $P C_{n}$, and comprise:

- Hog Features $\left(F_{H}\right)$ : this set includes $c_{w} \times c_{h} \times c_{b}$ features. Their values represent the information contained in a set of Histogram of Oriented Gradients (HOG) applied to the area surrounding the pore. We compute these features using the algorithm described in [46]. First we calculate the gradient module image $G_{M}(x, y)$ and the gradient phase image $G_{P}(x, y)$ of $I_{n}$. Then, we divide $I_{n}$ into $c_{w} \times c_{h}$ blocks. For each block, we quantize the orientation $\tilde{G}_{P}(x, y)$ into $c_{b}$ orientation bins. These bins are weighted by its magnitude $G_{M}(x, y)$. For each block, we calculate a histogram with $c_{b}$ orientations.

- Local Mean: $\left(F_{M}\right)$ : this feature indicates the mean gray level value of $I_{n}$.

- Local Variance $\left(F_{V}\right)$ : this feature indicates the standard deviation of the gray level value of $I_{n}$.

- Smoothness $\left(F_{S}\right)$ : this feature measures the relative smoothness of the intensity in $I_{n}$ as: $F_{S}=1-\frac{1}{\left(1+F_{V}\right)^{2}}$.

- Third moment $\left(F_{T}\right)$ : this feature measures the skewness of the histogram of $I_{n}$. It is calculated as: $F_{T}=\sum_{i=0}^{L-1}\left(z_{i}-\right.$ $\left.F_{M}\right)^{3} p\left(z_{i}\right)$, where $L$ indicates the number of intensity values, $z_{i}$ the intensity of pixel $i$ and $p(z)$ is the histogram of the intensity levels in $I_{n}$.

- Uniformity $\left(F_{U}\right)$ : this feature indicates the uniformity in $I_{n}$. It is computed as: $F_{U}=\sum_{i=0}^{L-1} p^{2}\left(z_{i}\right)$, where $L$ indicates the number of intensity values, $z_{i}$ the intensity of pixel $i$ and $p(z)$ is the histogram of the intensity levels in $I_{n}$.

- Entropy $\left(F_{E n}\right)$ : a measure of the randomness in $I_{n}$. Estimated as $F_{e}=-\sum_{i=0}^{L-1} p\left(z_{i}\right) \log _{2} p\left(z_{i}\right)$.

- Gray-level co-occurrence matrix features: this set contains 4 features that summarize the information contained in the Gray-level co-occurrence matrix, including homogeneity, energy correlation, and contrast.

- LBP features $\left(F_{L}\right)$ : this set includes 255 features related to the Regular Local Binary Patterns (LBP) [47].

We consider the problem of determining if a candidate point is a pore as a classification problem. This task is performed by assigning two different classes to the points extracted during the extraction of candidate points phase: $\{$ "pore"; "spurious"\}. In this work, we use classifiers based on feedforward neural networks.

During training, we determine the class of a candidate point $P C_{n}$ by considering its Euclidean distance $d_{n}$ to the closest real pore:

$$
\begin{array}{r}
d_{n}=\min \left(d\left(P C_{n}, P R_{j}\right)\right) ; \\
P R_{j} \in P R,
\end{array}
$$

where $d(\cdot)$ indicates the Euclidean distance and $P R$ is the set of real pores manually estimated by a group of expert supervisors. Then, if $d_{n}$ is smaller than a maximum acceptable distance $M_{d}$, the candidate point $P C_{n}$ is considered a "pore", otherwise, it is considered "spurious". The sets containing the pores and spurious points, based on the distance $M_{d}$, are defined as follows:

$$
\begin{aligned}
& \text { PORES }_{M_{d}}=\left\{P C_{n} \in P C: d_{n} \leq M_{d}\right\} ; \\
& \text { SPURIOUS }_{M_{d}}=P C-\text { PORES }_{M_{d}},
\end{aligned}
$$

where $M_{d}$ represents the maximum acceptable distance.

By adjusting $M_{d}$ we can adapt the behavior of the system. For instance, the choice of a very small distance $M_{d}$ can make the system more precise, although reducing the number of found pores. On the other hand, the increase of $M_{d}$ can increase the number of found pores, although reducing the precision of their estimated position.

\section{EXPERIMENTAL RESULTS}

\section{A. Setup and parameters}

The used setup is shown in Figure 2 and is composed by a commercial off-the-shelf color CCD camera and a green LED illumination. The distance from the camera to the LED is $\Delta_{L}=80 \mathrm{~mm}$, while the distance from the camera lens to the surface where the finger is placed is $165 \mathrm{~mm}$. The LED was placed at a distance $\Delta_{M}=100 \mathrm{~mm}$ to the surface where the finger is placed and inclined with an angle $\theta=75^{\circ}$ with respect to the camera, in order to provide an incident light enhancing the details of the sweat pores. The parameters of the camera used to capture the images are a shutter time $t_{s}=4 \mathrm{~ms}$, ISO 1600 and F16 aperture, chosen in order to acquire images without noise or motion blur. A macro lens with $100 \mathrm{~mm}$ focal length was used in order to capture the small details of the pores. The size of the captured images is $W \times H=5472 \times 3648$ pixels and describe an area with size $W \times H \approx 35 \times 25 \mathrm{~mm}$, resulting in images featuring a $\approx 3800$ ppi resolution.

\section{B. Database collection}

In order to test the validity of the proposed approach, we collected a database of 4 different fingerprints from 12 different people, for a total of 48 different fingerprints. Each fingerprint was captured separately 2 times, for a total of 96 images. The fingers were removed from the field of view of the camera after each acquisition, and then placed again.

After the acquisition, only the central region with size $W \times H=1500 \times 1000$ pixels, centered in the fingerprint core, was considered for the extraction of pores. Then, a group of three supervisors manually labeled the pores in each extracted region in order to establish the ground truth $P R$ for the experimental evaluations, for a total of 9,143 labeled pores. An example of the extracted ground truth in touchless 


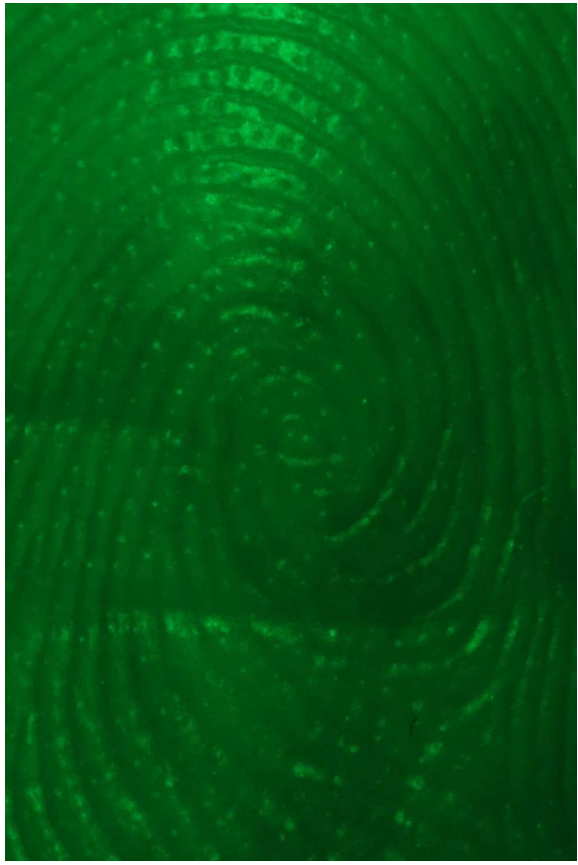

(a)

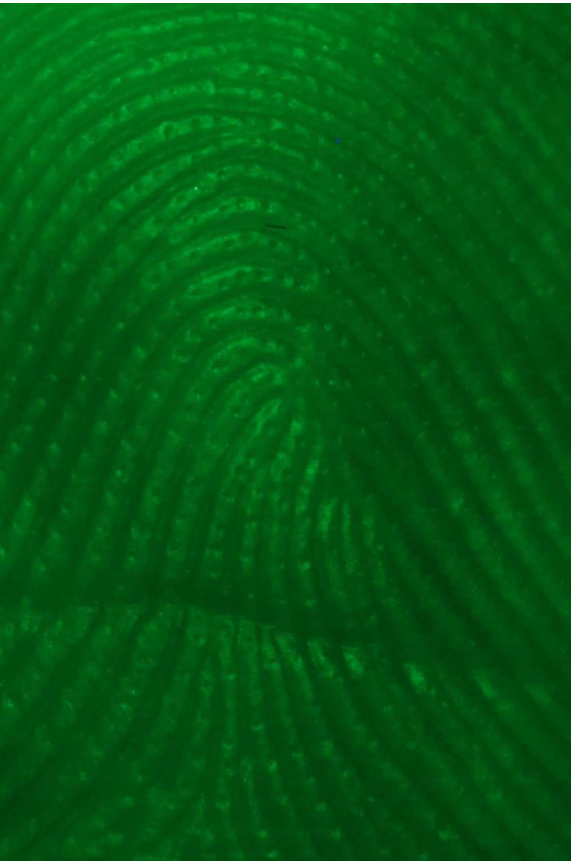

(b)

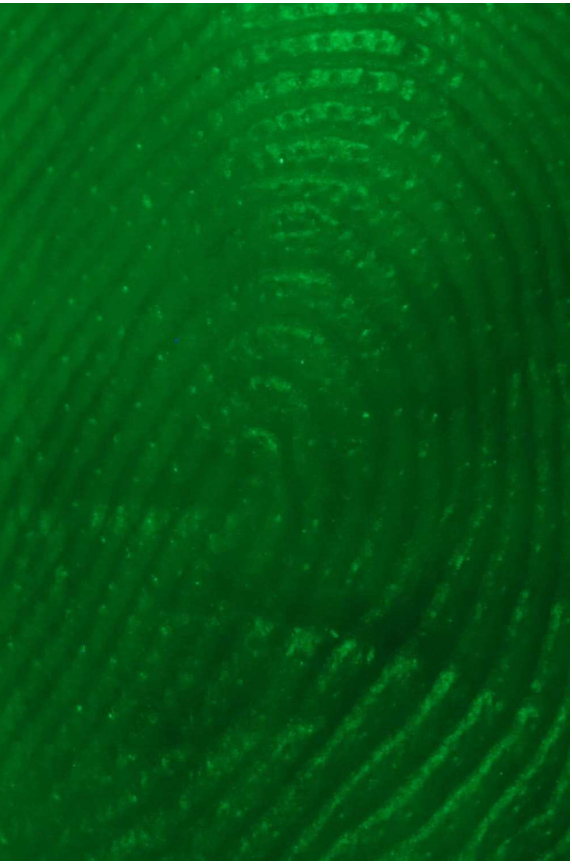

(c)

Fig. 4. Particulars of the central region, centered in the core, of the touchless fingerprint images captured from different individuals using the proposed approach. It is possible to observe the sweat pores present on the ridges.

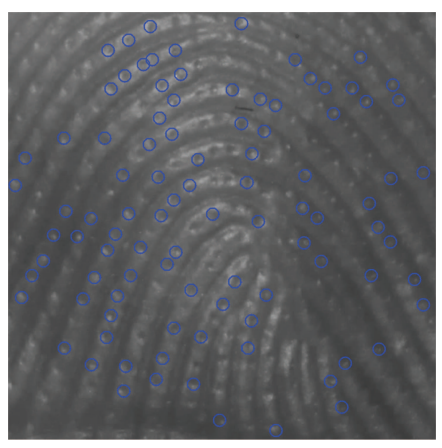

(a)

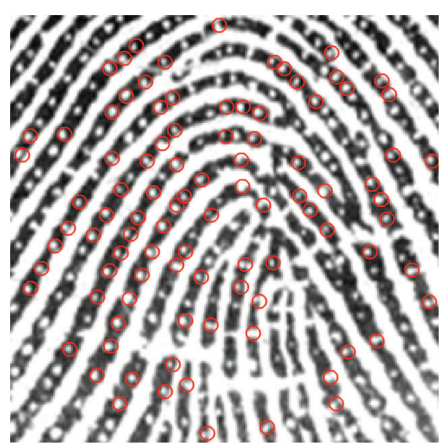

(b)

Fig. 5. Example of the ground truth extracted from a touchless sample captured using the proposed approach, and the corresponding touch-based image: (a) touchless sample; (b) touch-based image. It is possible to observe that approximately the same pores can be visible in both cases.

samples is shown in Figure 5 and compared with the ground truth extracted from the corresponding touch-based image. It is possible to observe that approximately the same pores can be visible in both cases.

Due to differences in the size and visibility of the pores, it was not possible to consider the fingerprints of every person: in some cases, the pores are not clearly visible in the captured images, and it is not possible to establish the ground truth for such images with sufficient confidence. A similar situation can happen also when extracting Level 2 details, since the fingerprint ridges are not always clearly visible [2]. For this reason, we treated the images in which the pores are not clearly visible as Failure To Enroll (FTE) situations. In particular, we discarded a total of 30 images for this reason. The results introduced in the following subsections consider the remaining
66 images, which contained a total of 26,977 candidate points.

\section{Experimental procedure}

For each image in the used database, we applied the proposed method for the estimation of the position of the candidate sweat pores $P C$, then we used computational intelligence techniques in order to compute a neural post-processing of the pores position, by assigning each candidate point in either the set PORES or SPURIOUS, according to Eq. 2. In particular, we used a Feed Forward Neural Network with one input layer, one hidden layer and one output layer. The input layer is composed by 61 nodes, equal to the number of used features, and the output layer is composed by 1 node, with a linear transfer function. We tested different number of nodes, with tan-sigmoidal transfer functions, in the hidden layer: 20,30, 50,70,100. A resilient back-propagation algorithm, using at most 400 epochs, was used to train the neural networks. We considered only neural networks with a single hidden layer, since they can be considered as universal approximators.

As a figure of merit, we considered the pore estimation accuracy as the proportion PTP of pores that lie at a distance $\leq d$ pixels of a real pore estimated manually, where $d$ is the maximum distance allowed in order to consider a pore as correctly estimated:

$$
\operatorname{PTP}(d)=\frac{|T P(d)|}{|P C|},
$$

where $P C$ is the set of candidate pores, $|\cdot|$ indicates the cardinality of the set, and $T P(d)=$ PORES $_{d}$, defined according to Eq. 2. 


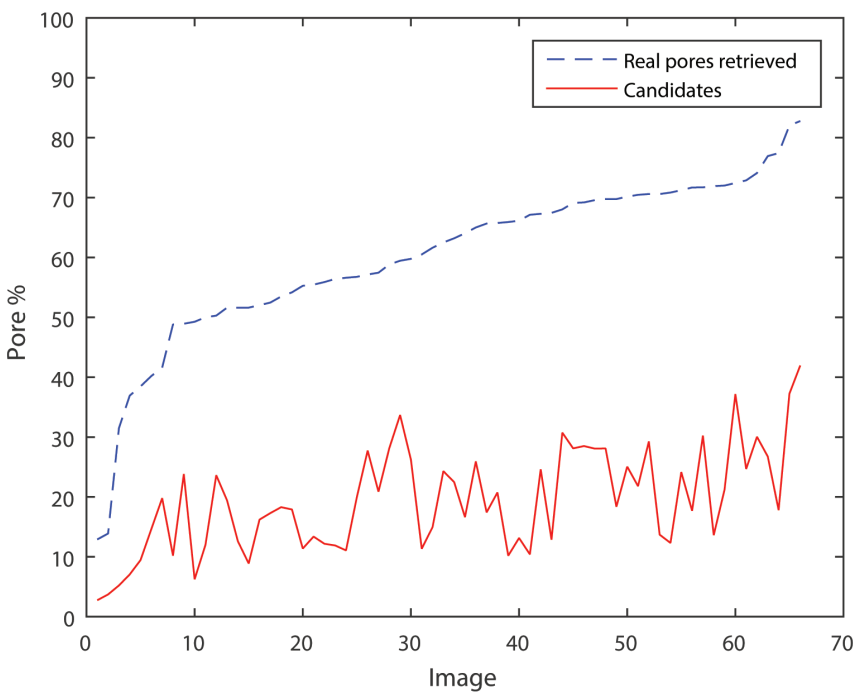

Fig. 6. Algorithm's error rates: the red line indicates the proportion of candidate points $P T P(r w / 4)$ found within the range of $r w / 4$ pixels from a real pore, where $r w=40$ pixels is the ridge width in the used images, while the dashed blue line indicates the amount of real pores retrieved by the algorithm within the range of $r w / 4$ pixels. The $X$ axis represents the number of images ordered by the amount of pores found by the supervisor. It is possible to observe that a neural post-processing step is necessary in order to increase the estimation accuracy.

\section{Results before applying computational intelligence}

In this section we study the results of the algorithm designed for the extraction of the candidate points, without applying the neural post-processing step described in Section III-C. Figure 7 presents the results of the algorithm, indicating the obtained average $P T P(d)$ for different values of $d$, for all the images in the dataset. Furthermore, Figure 6 illustrates the error rates of the algorithm for $\operatorname{PTP}(r w / 4)$, where $r w=40$ pixels is the average ridge width in the images of the used dataset. From the figures, it is possible to observe that a neural post-processing step is necessary in order to increase the estimation accuracy.

\section{E. Study of the performance of the neural post-processing step}

This section analyzes the performance of the neural network, used in the post-processing step, that classifies the candidate pore points in "pore" and "spurious" points. We compared the results obtained by the proposed neural classifier and other well-known techniques in the literature. In particular, we compared the performance of the proposed classification method with classifiers based on different learning techniques, such as k-Nearest Neighbor and Naive Bayes classifiers. Table I presents the obtained results calculated using a k-fold cross validation, with $k=10$ [48]. Moreover, Figure 7 shows the obtained average $P T P(d)$ for different values of $d$.

The obtained results show that the neural classifier outperformed the other methods on the considered dataset. In fact, simple classifiers did not obtain satisfactory accuracy on the considered problem, and classifiers able to approximate more complex functions, like a neural network with a large number of hidden units, obtained a significant reduction of the classification error. Furthermore, comparing the results obtained both with and without the refinement step using the
TABLE I

Results of the Neural Post-PRocessing STEP Using Different LEARNING TECHNIQUES.

\begin{tabular}{lcc}
\hline \hline \multirow{2}{*}{ Model } & \multicolumn{2}{c}{ Accuracy } \\
& Mean & Std \\
\hline kNN-1 & $76.0 \%$ & $1.6 \%$ \\
kNN-3 & $79.2 \%$ & $1.5 \%$ \\
Naive Bayes & $71.9 \%$ & $2.1 \%$ \\
FFNN-30 & $83.4 \%$ & $2.5 \%$ \\
\hline \hline
\end{tabular}

Note: FFNN-30 = Feed Forward Neural Network with 30 neurons in the hidden layer.

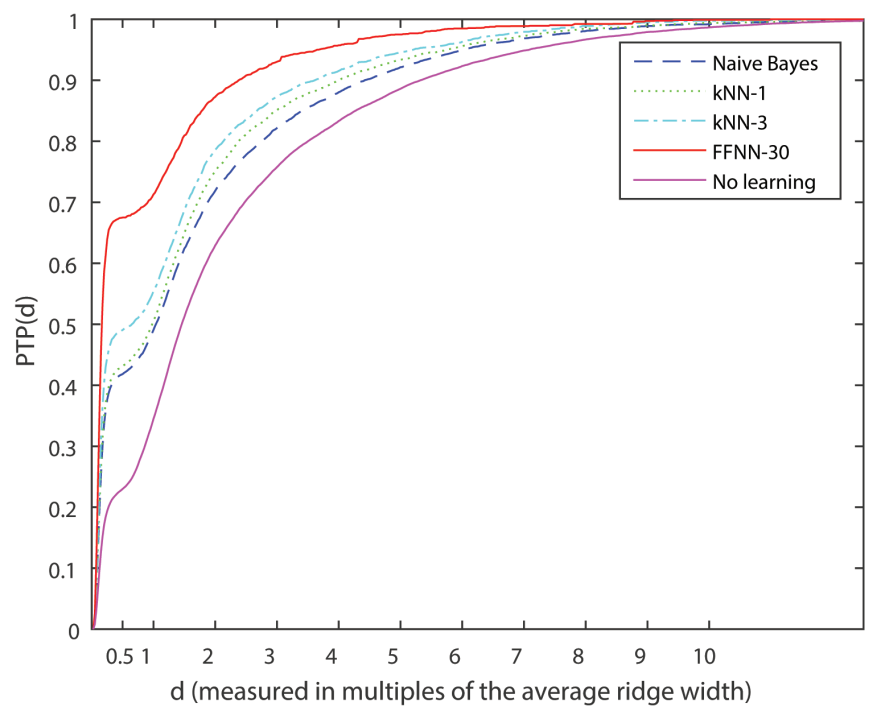

Fig. 7. Proportions of pores $P T P(d)$ that lie at a distance $<d$ of a real pore estimated manually, where $d$ is the maximum distance allowed in order to consider a pore as correctly estimated. The displayed curves present the results obtained before any learning technique is applied (magenta solid line), and after applying Naive Bayes (blue dashed line), kNN-1 (green dotted line), $\mathrm{kNN}-3$ (cyan dash-dot line) and FFNN-30 (red solid line). It is possible to observe that the use of neural networks allowed the greatest pore estimation accuracy.

neural network, it is possible to observe that the use of neural networks allows to increase the accuracy in the estimation of the position of the pores.

However, the analysis of which computational intelligence technique obtains the best results is out of the scope of this paper, since it is also dependent on the specific data of the studied application. Other computational intelligence techniques, like Support Vector Machines, could be analyzed in future research studies.

Moreover, Figure 8 shows for each image the number of extracted pores and $|T P(r w / 4)|$. Such numbers are sufficiently high to allow a recognition using the position of the pores for most images, since about $20-40$ pores can be used to assess the identity of an individual, as demonstrated by Jain et al. [25], thus the proposed approach shows the feasibility of a Level 3-based recognition using touchless fingerprint images.

\section{F. Computational speed}

We implemented the proposed method using Matlab, and run on an Intel Xeon $3.30 \mathrm{Ghz}$ CPU with $8 \mathrm{~GB}$ RAM. The used OS was Windows 7 Professional 64 bit. The average 


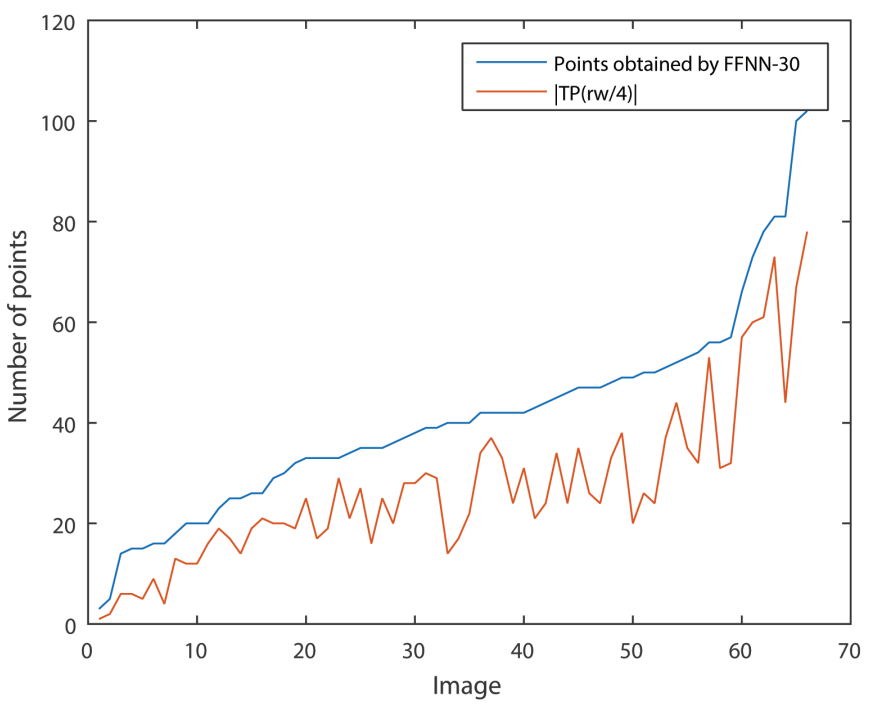

Fig. 8. Number of extracted pores and $|T P(r w / 4)|$ for each test image. Images are sorted in ascending order of number of extracted points. These numbers are sufficiently high to allow a recognition for most images, as demonstrated by Jain et al. [25].

time needed for extracting the candidate points was $6.43 \mathrm{~s}$ per image, while in average $0.01 \mathrm{~s}$ per image were needed to perform the neural post processing. However, the Matlab programming language is a prototypal non-optimized environment, and real-time processing speeds could be obtained if using, for example, a $\mathrm{C} / \mathrm{C}++$ implementation.

\section{CONCLUSiOnS AND FUTURE WORK}

In this work we proposed a novel method for the extraction of Level 3 features, in particular sweat pores, from fingerprint images captured using a touchless acquisition. The method is based on image processing algorithms and computational intelligence techniques. We applied the method on a database composed by 66 images captured from 12 different individuals. In order to establish the ground truth, 9,143 pores were manually labeled by three supervisors. The results showed the validity of the proposed approach, with the majority of pores correctly extracted, indicating that a touchless fingerprint recognition using Level 3 features is feasible.

Since the pores visible in touchless fingerprint images can have different characteristics according to the individual, the used illumination, and the position of the finger at the moment of the acquisition, it was not possible to design a single method able to extract the pores in every situation. For this reason, we studied a method based on image processing algorithms in order to extract a set of candidate points, and then we considered a set of features that could allow a computational intelligence classifier, using a set of examples, to learn and generalize the relation between the considered features and the presence of a real pore. Moreover, the use of neural techniques could allow the method to adapt to different cameras, resolutions, acquisition distances, and illumination conditions.

The proposed method could prove useful in future works for the design and implementation of biometric recognition algorithms able to work on Level 3 details in touchless images, in order to increase the accuracy of current touchless fingerprint recognition systems, or for the creation of innovative and more accurate antispoofing algorithms able to work on touchless fingerprint samples.

\section{REFERENCES}

[1] R. Donida Labati, A. Genovese, V. Piuri, and F. Scotti, "Measurement of the principal singular point in contact and contactless fingerprint images by using computational intelligence techniques," in Proc. of the IEEE Int. Conf. on Computational Intelligence for Measurement Systems and Applications, 2010, pp. 18-23.

[2] D. Maltoni, D. Maio, A. K. Jain, and S. Prabhakar, Handbook of Fingerprint Recognition, 2nd ed. Springer Publishing Company, Incorporated, 2009.

[3] D. Zhang, F. Liu, Q. Zhao, G. Lu, and N. Luo, "Selecting a reference high resolution for fingerprint recognition using minutiae and pores," IEEE Trans. on Instrumentation and Measurement, vol. 60, no. 3, pp. 863-871, 2011.

[4] T. Kwon and H. Moon, "Biometric authentication for border control applications," IEEE Trans. on Knowledge and Data Engineering, vol. 20, no. 8, pp. 1091-1096, 2008.

[5] R. Donida Labati, A. Genovese, E. Muñoz, V. Piuri, F. Scotti, and G. Sforza, "Automatic classification of acquisition problems affecting fingerprint images in automated border controls," in Proc. of the IEEE Workshop on Computational Intelligence in Biometrics and Identity Management, 2015.

[6] R. Donida Labati, A. Genovese, E. Muñoz, V. Piuri, F. Scotti, and G. Sforza, "Advanced design of automated border control gates: Biometric system techniques and research trends," in Proc. of the IEEE Int. Symp. on Systems Engineering, 2015, pp. 412-419.

[7] M. Yamazaki, D. Li, T. Isshiki, and H. Kunieda, "SIFT-based algorithm for fingerprint authentication on smartphone," in Proc. of the 6th Int. Conf. of Information and Communication Technology for Embedded Systems, 2015, pp. 1-5.

[8] W. Kang, X. Chen, and Q. Wu, "The biometric recognition on contactless multi-spectrum finger images," Infrared Physics \& Technology, vol. 68, pp. 19-27, 2015.

[9] F. Liu, D. Zhang, C. Song, and G. Lu, "Touchless multiview fingerprint acquisition and mosaicking," IEEE Trans. on Instrumentation and Measurement, vol. 62, no. 9, pp. 2492-2502, 2013.

[10] F. Liu, D. Zhang, and Z. Guo, "Distal-interphalangeal-crease-based user authentication system," IEEE Trans. on Information Forensics and Security, vol. 8, no. 9, pp. 1446-1455, 2013.

[11] R. Donida Labati, A. Genovese, V. Piuri, and F. Scotti, "A neural-based minutiae pair identification method for touch-less fingerprint images," in Proc. of the IEEE Workshop on Computational Intelligence in Biometrics and Identity Management, 2011, pp. 96-102.

[12] V. Piuri and F. Scotti, "Fingerprint biometrics via low-cost sensors and webcams," in Proc. of the 2nd IEEE Int. Conf. on Biometrics: Theory, Applications and Systems, 2008, pp. 1-6.

[13] F. Liu, D. Zhang, and L. Shen, "Study on novel curvature features for 3D fingerprint recognition," Neurocomputing, vol. 168, pp. 599-608, 2015.

[14] A. Kumar and C. Kwong, "Towards contactless, low-cost and accurate 3D fingerprint identification," IEEE Trans. on Pattern Analysis and Machine Intelligence, vol. 37, no. 3, pp. 681-696, 2015.

[15] R. Donida Labati, V. Piuri, and F. Scotti, Touchless Fingerprint Biometrics, ser. Security, Privacy and Trust. CRC Press, 2015.

[16] R. Donida Labati, A. Genovese, V. Piuri, and F. Scotti, "Touchless fingerprint biometrics: a survey on 2D and 3D technologies," Journal of Internet Technology, vol. 15, no. 3, pp. 325-332, 2014.

[17] R. Donida Labati, A. Genovese, V. Piuri, and F. Scotti, "Contactless fingerprint recognition: A neural approach for perspective and rotation effects reduction," in Proc. of the IEEE Workshop on Computational Intelligence in Biometrics and Identity Management, 2013, pp. 22-30.

[18] R. Donida Labati, A. Genovese, V. Piuri, and F. Scotti, "Accurate 3D fingerprint virtual environment for biometric technology evaluations and experiment design," in Proc. of the IEEE Int. Conf. on Computational Intelligence and Virtual Environments for Measurement Systems and Applications, 2013, pp. 43-48.

[19] R. Donida Labati, A. Genovese, V. Piuri, and F. Scotti, "Two-view contactless fingerprint acquisition systems: A case study for clay artworks," in Proc. of the IEEE Workshop on Biometric Measurements and Systems for Security and Medical Applications, 2012, pp. 1-8. 
[20] R. Donida Labati, A. Genovese, V. Piuri, and F. Scotti, "Toward unconstrained fingerprint recognition: A fully touchless 3-D system based on two views on the move," IEEE Trans. on Systems, Man, and Cybernetics: Systems, vol. 46, no. 2, pp. 202-219, 2016.

[21] K. Tiwari and P. Gupta, "A touch-less fingerphoto recognition system for mobile hand-held devices," in Proc. of the 2015 Int. Conf. on Biometrics, 2015, pp. 151-156.

[22] R. Raghavendra, C. Busch, and B. Yang, "Scaling-robust fingerprint verification with smartphone camera in real-life scenarios," in Proc. of the IEEE Sixth Int. Conf. on Biometrics: Theory, Applications and Systems, 2013, pp. 1-8.

[23] B. Yang, G. Li, and C. Busch, "Qualifying fingerprint samples captured by smartphone cameras," in Proc. of the 20th IEEE Int. Conf. on Image Processing, 2013, pp. 4161-4165.

[24] M. Varges da Silva, A. N. Marana, and A. A. Paulino, "On the importance of using high resolution images, third level features and sequence of images for fingerprint spoof detection," in Proc. of the IEEE Int. Conf. on Acoustics, Speech and Signal Processing, 2015, pp. 18071811.

[25] A. K. Jain, Y. Chen, and M. Demirkus, "Pores and ridges: Highresolution fingerprint matching using level 3 features," IEEE Trans. on Pattern Analysis and Machine Intelligence, vol. 29, no. 1, pp. 15-27, 2007.

[26] A. Abhyankar and S. Schuckers, "Towards integrating level-3 features with perspiration pattern for robust fingerprint recognition," in Proc. of the 17th IEEE Int. Conf. on Image Processing, 2010, pp. 3085-3088.

[27] S. Malathi, S. U. Maheswari, and C. Meena, "Fingerprint pore extraction based on marker controlled watershed segmentation," in Proc. of the 2nd Int. Conf. on Computer and Automation Engineering, vol. 3, 2010, pp. 337-340.

[28] R. F. da Silva Teixeira and N. J. Leite, "On adaptive fingerprint pore extraction," in Image Analysis and Recognition, ser. Lecture Notes in Computer Science, M. Kamel and A. Campilho, Eds. Springer Berlin Heidelberg, 2013, vol. 7950, pp. 72-79.

[29] Q. Zhao, L. Zhang, D. Zhang, and N. Luo, "Direct pore matching for fingerprint recognition," in Advances in Biometrics, ser. Lecture Notes in Computer Science, M. Tistarelli and M. Nixon, Eds. Springer Berlin Heidelberg, 2009, vol. 5558, pp. 597-606.

[30] S. Malathi and C. Meena, "An efficient method for partial fingerprint recognition based on local binary pattern," in Proc. of the IEEE Int. Conf. on Communication Control and Computing Technologies, 2010, pp. $569-572$.

[31] J. Cui, M.-S. Ra, and W.-Y. Kim, "Fingerprint pore matching method using polar histogram," in Proc. of the 18th IEEE Int. Symp. on Consumer Electronics, 2014, pp. 1-2.

[32] P. Agrawal, R. Kapoor, and S. Agrawal, "A hybrid partial fingerprint matching algorithm for estimation of Equal Error Rate," in Proc. of the Int. Conf. on Advanced Communication Control and Computing Technologies), 2014, pp. 1295-1299.

[33] S. Malathi and C. Meena, "Improved partial fingerprint matching based on score level fusion using pore and SIFT features," in Proc. of the Int. Conf. on Process Automation, Control and Computing, 2011, pp. 1-4.

[34] Y. Chen and A. K. Jain, "Dots and incipients: Extended features for partial fingerprint matching," in Proc. of the Biometrics Symp., 2007, pp. 1-6.

[35] Q. Zhao and A. K. Jain, "On the utility of extended fingerprint features: A study on pores," in Proc. of the IEEE Computer Society Conf. on Computer Vision and Pattern Recognition Workshops, 2010, pp. 9-16.

[36] D. Li, H. Kunieda, S. Kumpituck, and T. Isshiki, "Online detection of spoof fingers for smartphone-based applications," in Proc. of the IEEE 7th Int. Symp. on High Performance Computing and Communications, 2015, pp. 1292-1297.

[37] M. Varges da Silva, A. N. Marana, and A. A. Paulino, "On the importance of using high resolution images, third level features and sequence of images for fingerprint spoof detection," in Proc. of the IEEE Int. Conf. on Acoustics, Speech and Signal Processing, 2015, pp. 18071811.

[38] M. Lu, Z. Chen, and W. Sheng, "Fingerprint liveness detection based on pore analysis," in Biometric Recognition, ser. Lecture Notes in Computer Science, J. Yang, J. Yang, Z. Sun, S. Shan, W. Zheng, and J. Feng, Eds. Springer International Publishing, 2015, vol. 9428, pp. 233-240.

[39] P. Johnson and S. Schuckers, "Fingerprint pore characteristics for liveness detection," in Proc. of the Int. Conf. of the Biometrics Special Interest Group, 2014, pp. 1-8.

[40] G. L. Marcialis, F. Roli, and A. Tidu, "Analysis of fingerprint pores for vitality detection," in Proc. of the 20th Int. Conf. on Pattern Recognition, 2010, pp. 1289-1292.

[41] M. Espinoza and C. Champod, "Using the number of pores on fingerprint images to detect spoofing attacks," in Proc. of the Int. Conf. on HandBased Biometrics, 2011, pp. 1-5.

[42] Z. Saquib, S. Soni, and R. Vig, "Sweat pores-based (level 3) novel fingerprint quality estimation," in Proc. of the 3rd IEEE Int. Conf. on Computer Science and Information Technology, 2010, pp. 525-531.

[43] M. Vatsa, R. Singh, A. Noore, and S. K. Singh, "Quality induced fingerprint identification using extended feature set," in Proc. of the 2nd IEEE Int. Conf. on Biometrics: Theory, Applications and Systems, 2008, pp. 1-6.

[44] M. Pamplona Segundo and R. de Paula Lemes, "Pore-based ridge reconstruction for fingerprint recognition," in Proc. of the IEEE Conf. on Computer Vision and Pattern Recognition Workshops, 2015, pp. 128133.

[45] L. Wang, R. H. Abd El-Maksoud, J. M. Sasian, and V. S. Valencia, "Illumination scheme for high-contrast, contactless fingerprint images," Proc. SPIE, vol. 7429, pp. 742 911-742 911-7, 2009.

[46] D. G. Lowe, "Distinctive image features from scale-invariant keypoints," Int. Journ. of Computer Vision, vol. 60, no. 2, pp. 91-110, 2004.

[47] T. Ojala, M. Pietikäinen, and D. Harwood, "A comparative study of texture measures with classification based on featured distributions," Pattern recognition, vol. 29, no. 1, pp. 51-59, 1996.

[48] R. O. Duda, P. E. Hart, and D. G. Stork, Pattern Classification (2nd Edition), 2nd ed. Wiley-Interscience, 2001. 